\title{
Obediencia institucional en el laboratorio
}

\section{J. M. Fernández Dols, G. J. García González, J. C. Gómez Ramírez, T. Martínez Díaz y R. Sanz Pérez}

Universidad Autónoma de Madrid

\section{INTRODUCCION}

Este trabajo pretende estudiar un tópico de la Psicología Social Experimental: aquel que se refiere a la conformidad. Afinando más en la elección de un área de intereses, puede decirse que la investigación se centra en dos paradigmas experimentales: el modelo de Milgram para el estudio de la "Obediencia a la autoridad" y el de Darley y Latané sobre "altruísmo en presencia de otros participantes" (Milgram, 1963, 1965, 1974; Darley-Latané, 1968 a 1969).

Como se sabe, en el experimento de Milgram se ordena a los $S$ que administren descargas, cada vez más altas -hasta llegar a los 450 voltios-, a una supuesta víctima que falla en un ejercicio de memorización; el porcentaje de sujetos obedientes, incluso interactuando con una víctima que se resistía de diversos modos a sufrir el castigo, fue inesperadamente alto. Darley y Latané observan cómo la presencia de algún observador en una situación experimental inhibe la conducta de ayuda de los sujetos para'socorrer a la experimentadora, que parece haberse dislocado un tobillo en un lugar próximo, o para avisar de que a un supuesto compañero de experiencia le ha dádo un ataque (vid., para una descripción y discusión 'de estos trabajos en castellano Torregrosa, 1974, pág. 261, Secord y Backman, 1976, págs. 322 y siguientes, entre otros) (1).

La elección de ambos paradigmas responde a varias razones. Puede afirmarse que, tanto en la experiencia de Milgram como en las de Darley y Latané, se establecieron situaciones experimentales que poseen, en si mismas, una cierta relevancia ecológica. En el laboratorio, un gran número de sujetos obedecian a órdenes "no humanitarias» o sencillamente se inhibían ante la solicitud de ayuda por parte de otro -en concreto una mujer herida o un joven con un ataque-. Es importante recalcar "en el laboratorio", porque cuando se realizaron experimentos de campo sobre el tema, por ejemplo, conducta de ayuda a supuestos enfermos o borrachos en el Metro de Nueva York, los resultados no concordaron con los de los trabajos de laboratorio: los $S$ tendieron a ayudar a "enfermos" y "borrachos» fingidos en gran número

(1) Para una descripción más detallada y todo tipo de cuestiones sobre este artículo, escríbase a J. M. Fernández Dols. Dto. de Ecopsicología, Facultad de Filosofía y Letras de la Universidad Autónoma de Madrid. 
de ocasiones, a pesar de que el número de personas en el vagón era alto, lo que favorecía una supuesta difusión de la responsabilidad, por la que se habian explicado los resultados de los experimentos comentados anteriormente ( $\mathrm{Pi}$ lavin et al., 1969).

Parece, pues, que existieron unas conductas éticas o valorativamente anormales ligadas a las situaciones "de laboratorio». Es posible que, como afirmó Mixon (1979), las conductas de los sujetos de estos experimentos no fueran tan inesperadas como Milgram dio a entender en su trabajo - comparando los resultados, i. e., el porcentaje de sujetos obedientes, con las predicciones de diversos grupos de "expertos": psiquiatras, estudiantes de psicología, etc.- - Sin embargo, lo "chocante" de los resultados de estos trabajos no pierde un ápice de efectividad con tal observación, ya que lo que en realidad se plantea en las experiencias sobre conformidad es su referencia a un modelo de conducta, un ideal semejante a aquel que nos hace hablar de salud mental como un máximo de independencia, productividad y satisfacción del individuo. De ahí el término "valorativamente anormal» utilizado anteriormente.

El laboratorio como "establecimiento" ("setting") (Barker, 1968) cobra así interés por sí mismo y permite no tener en cuenta algunas de las prevenciones clásicas hacia la validez del trabajo de laboratorio (vid., por ejemplo, Orne, 1962): el "laboratorio" sería aquí un establecimiento de gran importancia en nuestra vida social, y el estudio de la conducta dentro de él puede proporcionar inferencias de gran interés. Se puede afirmar que un "laboratorio" posee una gran importancia en tanto sería el establecimiento característico de la ciencia y los científicos; es, por tanto, importante conocer algunas pautas conductuales que caracterizan tal establecimiento (behavioral setting).

En la presente investigación puede decirse que se realiza un experimento con una validez ecológica elevada en tanto que el laboratorio no pretende extrapolar sus resultados a otros contextos. Dicho simplemente, el «laboratorio no puede ser otra cosa que un laboratorio".

Planteadas así las cuestiones, se realizó una experiencia en la que se estudiaron las reacciones de los sujetos ante una situación en la que simultáneamente:

\section{- según el paradigma de Milgram se exige obediencia; \\ - según el paradigma de Darley-La- tané se exige conducta de ayuda.}

Se trata de recoger en un solo diseno las dos consecuencias más llamativas de ambos paradigmas: un gran porcentaje de sujetos "no ayudan" u "obedecen acríticamente». Ambas quedan operacionalizadas en una sola conducta, con lo que es posible aventurar que, tanto en un caso como en otro, se puede localizar un factor común: un marco referencial (behavioral setting) considerado molarmente que se utiliza como variable independiente.

Como variable dependiente se considera una conducta observable del sujeto de experimentación que puede denominarse "obediencia a la autoridad" o "no existencia de conductas de ayuda», pero que, además, supone la adaptación a un rol regulado por unas reglas interactivas definidas por el marco situacional (V.I.).

\section{METODO}

Sujetos: 28 sujetos seleccionados al azar en base al fichero de alumnos de primero de Psicología de la Universidad Autónoma de Madrid, distribuidos en dos grupos aleatoriamente:

a) un grupo de control (dos varones, ocho mujeres) (15-2-80);

b) un grupo experimental (tres varones, doce mujeres) . (18-2-80, 22-2-80).

Diseño e instrumentación: Los $\dot{S}$ eran, en ambos casos, introducidos en una 


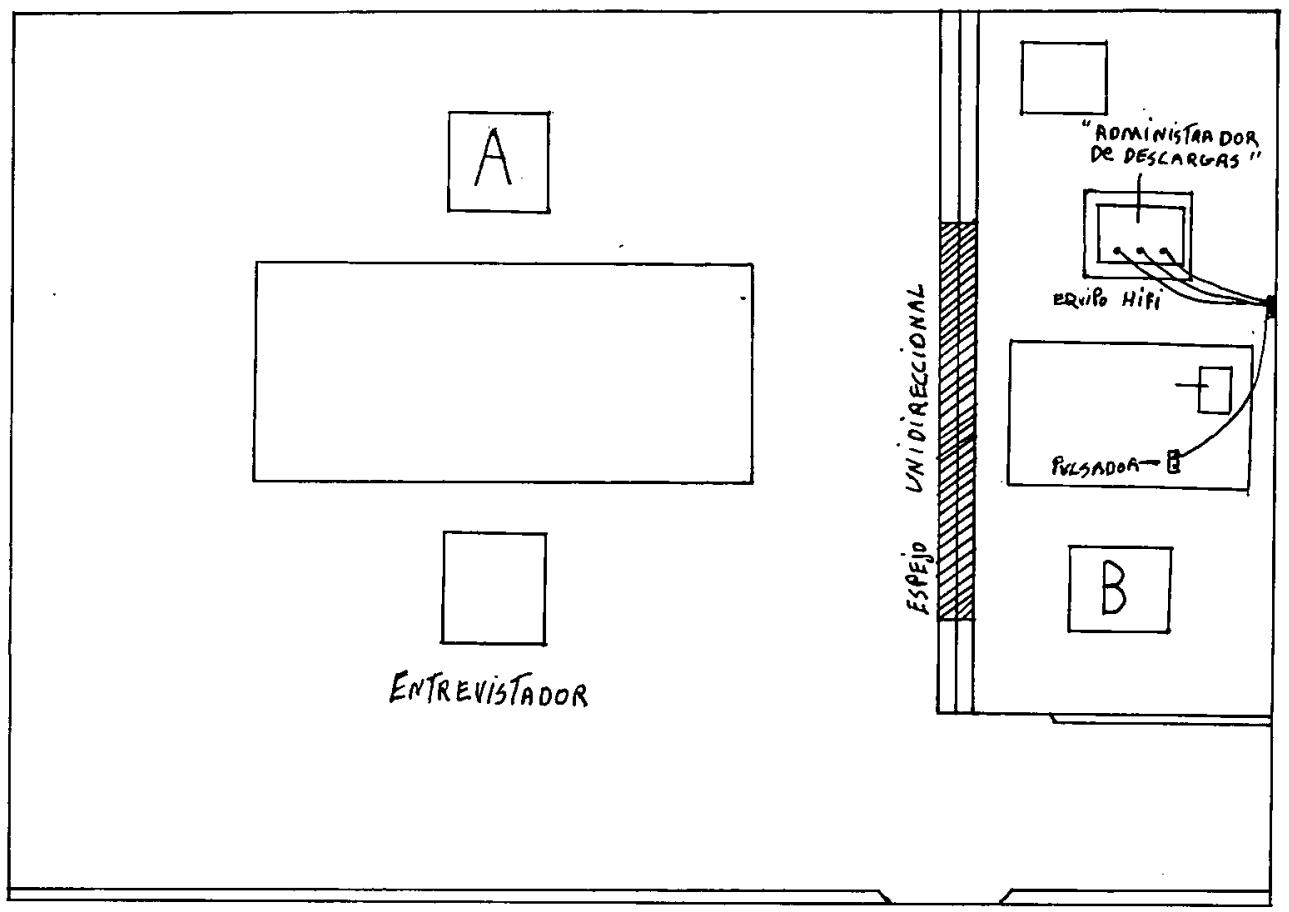

amplia sala con un ostensible espejo unidireccional (vid. plano), y debían permanecer sentados frente a una mesa - posición $\bar{A}$ - aprendiendo una lista de palabras.

En el caso del grupo de control, la experiencia se desarrollaba sin incidentes y se justificaba la presencia de dos personas en la cabina mediante las siguientes instrucciones:

"Este es un experimento sobre recuerdo mediante conceptualización y memorización visual. En nuestro experimento deberás memorizar unas palabras y luego reconocerlas entre otras que se te irán presentando en silencio, siempre por escrito. El reconocimiento de las palabras se producirá en la cabina de aquí al lado... Como ves, deberás sentarte en esta silla y apretar el botón negro si reconoces la palabra presentada en las láminas y el rojo si no la reconoces. Ahora vuelve a la mesa y comienza a memorizar tu lista; yo me quedaré aquí con mi compañero para registrar con un fotómetro y un fonómetro los posibles ruidos o cambios bruscos del ambiente luminoso que puedan perjudicar tu rendimiento. Tienes veinte minutos; por favor, no escribas nada y concéntrate en tu tarea. A partir de ahora debe guardarse absoluto silencion.

Los $S$ permanecían en tal situación 17 minutos y luego se les citaba, con la excusa de que se trataba de una prueba de memoria a largo plazo, para dos dias más tarde, tras comprobar que la situación no les había resultado in. verosimil.

En el caso del grupo experimental, los $S$ se encontraban, nada más entrar. a una muchacha que les cedía su sitio (el asiento $A$ ), como si terminase en ese momento de memorizar las palabras, y pasaba a ocupar el asiento B, en la cabina de control. Se le leían al sujeto las siguientes instrucciones:

"Este es un experimento sobre recuerdo mediante conceptualización y memorización visual. Un aspecto poco conocido de esta área es el papel de los castigos en el aprendizaje. En nuestro experimento deberás memorizar unas palabras y luego reconocerlas entre otras que se te irán presentando en 
silencio, siempre por escrito. Cuando se produzcan errores te administraremos un castigo, progresivamente mayor. El reconocimiento de las palabras se producirá en la cabina de aquí al lado... Como ves, deberás sentarte en esta silla (las correas son para evitar golpes por movimientos bruscos y fijar los electrodos). Deberás apretar el botón negro si no reconoces la palabra presentada en las láminas y el rojo si la reconoces. En caso de error se administrará un pequeño "calambre" de intensidad progresivamente mayor, aunque no llegará a producirte ninguna lesión. Mira, por ejemplo, como es el primer calambre... bien, esta demostración ha servido para que tu compañera recuerde su tarea; luego te la recordaremos como a ella. Ahora vuelve a la mesa y comienza a memorizar tu lista; yo me quedaré aquí. Tienes veinte minutos. Por favor, no escribas nada y concéntrate en tu tarea. A partir de ahora debe guardarse absoluto silencion.

Dentro de la cabina, un experimentador con bata blanca y el entrevista- maciones proseguían "in crescendo" hasta que en el minuto 15 y 29 segundos, sólo se oían gemidos (vid. trans. cripción).

Por lo demás, a partir del minuto 10 , los gritos se acompañaban de patadas.

Tras la prueba, los $S$ pasaban a ser entrevistados: se les explicaba la situación, pidiéndoles disculpas y cuestionándoles sobre sus experiencias durante la prueba. Por último, eran citados para la misma hora y día que el grupo de control (salvo en los $3 S$ experimentales del día 22).

Tal día y hora (miércoles 20 a las 14 horas) se convocó una última reunión en la que se comentó con todos los $S$ la experiencia y se aclararon dudas.

\section{RESULTADOS}

Se consideraron tres tipos de respuestas:

1. El sujeto no se levanta de la silla hasta que concluyen los 17 minutos de espera ( «no intervención»).

\section{Cuadro I. Transcripción abreviada de los quejidos de la víctima}
$1,39 \ldots$ uf
$3,12 \ldots$ chico, que duele mucho, ¿eh?
$5,13 \ldots$ ay, ay, que no sigo
$6,44 \ldots$ me niego a seguir con esto
$7,23 \ldots$ ay, ay, por favor, de verdad, que no lo puedo aguantar, que no pue- do aguantar este dolor
$9,13 \ldots$ me voy, que me voy, de verdad, esto no lo aguanto más. Ay, por favor dejadme irme, que yo no me creía que esto era así
$13,17 \ldots$ ay, soltadme, por favor
$15,29 \ldots$ jah!, ¡ah!

dor, le mostraban al sujeto el asiento $\mathrm{B}$-con correas- $\mathrm{y}$ un presunto administrador de descargas; para dar mayor verosimilitud a la situación, el sujeto recibía una descarga de $4 \mathrm{mi}$ liamperios. Luego volvía a su sitio (asiento A), y la cabina se cerraba. Transcurridos 1 minuto y 39 segundos se oían las primeras quejas, cada vez más intensas (se trataba de una grabación de alta fidelidad); a los tres minutos y doce segundos, la víctima afirmaba por primera vez que las descargas le dolían mucho, y a los cinco minutos y trece segundos, que no quería proseguir el experimento. Luego ambas afir-
1. El sujeto se levanta antes de que concluyan los 17 minutos:

2.1. Para irse ("huida sin intervención").

2.2. Para entrar en la cabina («intervención").

En el grupo de control todas las conductas se clasificaron en la categoría de «no intervención». En el grupo experimental, los resultados fueron como sigue: ll $S$ en "no intervención", de los cuales nueve fueron mujeres y dos varones $(73 \%) ; 2 S$ en whuida sin intervención" ambos mujeres (13,3\%); por último, $2 S$ en "intervención» un varón y una mujer $(13,3 \%)$. 
Si aplicamos la prueba de probabilidad exacta de Fisher no se aprecian diferencias entre las conductas del gru- po de control y las del grupo experimental:

Cuadro II

no intervención

y huida sin intervención intervención

0

2

$\begin{array}{lll}\text { Grupo Control } & 10 & 0 \\ \text { Grupo Experimental } & 13 & 2\end{array}$

no intervención

huida e intervención

Grupo Control

Grupo Experimental
10

11
0

4
En el segundo caso, p es .11, valor que no permite aceptar $\mathrm{H}_{1}$ con $=.10$ (se acepta $\mathrm{H}_{0}$ : no hay diferencias en las proporciones de rechazo). En el primer caso la probabilidad resultante es .34 , con lo que la similitud entre las proporciones de ambos grupos es aún mayor.

Por lo demás, cabe indicar que los sujetos que rompieron la rutina experimental lo hicieron a los 7 minutos y 10 segundos y los 13 minutos (en los casos de "huida sin intervención") y a los 14 minutos y 15 segundos y a los 15 minutos (en los casos de «intervención»).

\section{DISCUSION}

En primer lugar, llama la atención la similitud entre los porcentajes de "no intervención" obtenidos $(73 \%)$, y los resultados de Darley y Latané (aproximadamente un $80 \%$ en la condición en la que el individuo estaba acompanado) y de Milgram en el diseño que más se aproxima al nuestro (feed-back verbal de la víctima: un $62,5 \%$ de obedientes). Ello puede hacer pensar que el fenómeno reproducido en este experimento es semejante al estudiado en tales casos, al menos desde un punto de vista cuantitativo.

Respecto a los aspectos cualitativos, las respuestas proporcionadas en la entrevista postexperimental fueron categorizadas en los siguientes apartados:

a) S que aceptaron la situación y asumieron su papel de futuras victimas con mayor o menor aprensión.

b) $S$ que trataron de minimizar el contenido de los gritos apelando a una extrema sensibilidad de la víctima $\mathrm{y} / \mathrm{o}$ al hecho de que los experimentadores serían sancionados si realmente dañasen a la víctima.

c) $S$ que ignoraron las quejas de la víctima o no las consideraron graves en base al supuesto de que a ellos no les podría ocurrir lo mismo, ya que su nivel de rendimiento sería superior.

d) $S$ que manifestaron experimentar una sensación de irrealidad. No se apuntaron fallos en la simulación, sino que ésta se consideraba «increible" por su gravedad.

Tales tipos de respuestas pueden asemejarse a las registradas por DarleyLatané y Milgram en sus entrevistas postexperimentales. En la segunda categoría se detectaría el proceso de difusión de la responsabilidad que acuñan Darley-Latané y que también utiliza Milgram, i.e. una pérdida de la autonomía en base a la apelación a responsabilidades de otros $S$. En la primera y tercera categoría se localizarían casos de conformidad extrema; es especialmente in- 
teresante la tercera categoría, que afecta a dos sujetos, en la que - además de aceptación pasiva- quizás intervengan variables como autoestima y estilo cognoscitivo, hipótesis a comprobar en futuras experiencias. En cuanto al cuarto tipo de respuestas, éste se ajustaría al concepto de "mala percepción de la realidad" de Darley-Latané y a una ruptura característica de los esquemas de acción del sujeto cuando éste se halla en situaciones que rompen con 'sus marcos de referencia cognoscitivos.

En todo caso, se haya o no creído la situación, lo cierto es que los $S$ siguieron, en su inmensa mayoría, respetando la situación - «behavioral setting"establecida. Es justamente ese fenómeno, esa conformidad hacia la propia situación experimental, por desagradable o absurda que sea, lo que -en cualquier caso- resulta indudable, al menos en las poblaciones consideradas.

Así, la polémica Orne-Milgram (2) perdería gran parte de su sentido: Orne y Holland defienden que el sujeto obedece, no porque pierda su autonomía ante la autoridad, sino porque considera que el laboratorio implica forzosamente una garantía de seguridad -el sujeto "sabe" que no pasa "nada malo" porque "no puede pasar»-. Por su parte, Milgram defiende la verosimilitud de la situación y su posible extrapolación a situaciones más genéricas - por ejemplo, los campos de exterminio nazis-.

En la presente investigación, se piensa que, considerando a la variable independiente como un establecimiento conductual sujeto a observación, en concreto la situación de laboratorio, se llega a un punto de confluencia de ambas posturas; es cierto, como dicen Orne y Holland que el laboratorio sitúa al sujeto en una actitud especial, pero si se sustituye la denominación "obediencia a la autoridad" por la de «obediencia al laboratorio», el problema sigue teniendo la trascendencia que defiende Milgram, aunque quizá desde una nueva perspectiva que tenga en cuenta los aspectos ecológicos de la situación: el laboratorio como «establecimiento conductual" provoca conductas «aberrantes». El laboratorio tiene una significación social importante, ligada a la representación social de la ciencia (Herzlich, 1972), así pues, no es en absoluto irrelevante subrayar esta obediencia institucional en el establecimiento científico por excelencia. Ahora bien: la cuestión de si tal "obediencia a la autoridad", según la terminología de Milgram, aparece en otros establecimientos sociales, exigirá la consiguiente taxonomía de situaciones relevantes en la que no siempre, necesariamente, se detectará tal "obediencia".

\section{CONCLUSION}

Numerosos investigadores han discutido la validez experimental y las implicaciones éticas del trabajo de Milgram. En último término parece que unos y otros están de acuerdo en que:

1. La situación es, al menos, desagradable.

2. La situación es inusual, y en sus planteamientos exige conductas - como afirma Milgram- o la representación de conductas - como afirmarían algunos de sus críticosque van contra ciertos valores comúnmente aceptados y respetados.

3. Los sujetos se acomodan en porcentajes más que notables a la situación.

Los resultados especificados en este informe se ajustan perfectamente a estas investigaciones. No es casual, por lo demás, que se insista en el término "situación", ya que parece que lo más relevante de los paradigmas en los que

(2) Para-una visión general de la polémica que siguió a la publicación de los trabajos de Milgram, vid. Miller 1972, cap. 2. 
se integra este experimento es la situación, el establecimiento conductual: el laboratorio elicitaría pautas de conducta extremadamente conformistas.

Por lo demás, los resultados apuntan a la necesidad de ampliar esta línea de inveștigación en diversas direcciones, por ejemplo:

- ampliando la relevancia de las muestras.

- controlando la interacción entre los resultados y ciertas variables: sexo de los $S$ y de la víctima, actitudes de los $S$, etc.

- estableciendo una taxonomía de aquellos "settings" en los que sería especialmente relevante el estudio de la conformidad.

- en el caso concreto del laboratorio, estableciendo la relación entre la representación social de la ciencia y constructos como conformidad y control social.
- considerando la importancia del contexto académico - aspecto apuntado por Milgram- en el laboratorio como establecimiento conductual.

Son estos, indudablemente, temas demasiado gruesos para este informe, cuyo propósito sería abrir una línea de investigación muy característica en Psicología Ecológica (Wicker, 1979) -el estudio del setting - en el marco de los paradigmas experimentales de la Psicología Social y, en cuanto al constructo "conformidad" seguir una perspectiva interaccionista como la marcada por Hollander (1975) cuando afirmaba:

"... La conformidad debe considerarse contextualmente como resultado de la interacción de la persona con un grupo en una situación social específica; asi pues, no se trata de un atributo del individuo, sino más bien de un concepto relacional».

\section{Resumen}

La presente investigación consistió en una réplica de los paradigmas experimentales de Milgram y Darley y Latané sobre conformidad y conducta de ayuda. Se utilizaron $28 S$ divididos en un grupo de control y uno experimental. La V.I. era la presencia o ausencia de una supuesta víctima que, en una estancia contigua, recibía descargas eléctricas contra su voluntad. La V.D. estudiada era la obediencia o no a las instruc. ciones del experimentador por parte de los $S$ en ambas situaciones. Los resultados confirman los de anteriores trabajos al observarse altos porcentajes de conductas conformistas. Por último se discuten tales datos en base a su relevancia ecológica.

\section{Summary}

The present research is a replication of the experimental paradigms of Milgram and Darley y Latané on conformity and Assistance Behavior. 28 subjects wete used. They were divided in a control and an experimental group. The V.D. was the pressence or ausence of a supposed victim who received electric shocks, against his volunty, in a conciguons room. The D.V. was the obedience or not of the instructions of the expe. rimenter by the subjects in botn conditions. The results confirm the findings of other investigations, showing high proportions of conformist behaviors. Finally, the findings are discussed from the point of view of their ecological relevance. 


\section{Estudios}

\section{Referencias}

BARKER, R. G.: Ecological Psychology: Concepts and methods for studyng the environment of buman behavior. Standford, Standford University Press, 1968.

DARLEY, J. M; LATANE, B.: Bystander intervention in emergencies: Diffusion of responsability. Joumal of Personality and Social Psychology, 1968, 8, 377-383.

HERZLICH, C.: La representation social en Moscovici, S. (dir.). Introduction a lo psychologie sociale. París, Larousse, 1972.

HOLLANDER; E. P.: Independence, Conformity and Civil Liberties: Some Implications from Social Psychological Research. Journal of Social Issues, 1975, 31, 275-282.

LATANE, B.; DARLEY, B.: Group inhibition of bystander intervention. Journal of Personality and Social Psychology, 1968, 16, 215-221.

LATANE, B.; RODIN, J.: A lady in distress: Inhibiting effects of friends and strangers on bystander intervention. Journal of Experimental Social Psychology, 1969, S, 189-202.

MILGRAM, S.: Behavioral study of obedience. Joumal of A bnormal and Social Psychology, 1963, 67, $371-378$.

MILGRAM, S.: Some conditions of obedience and disobedience to authority. Human Relations, 1965, 18, 57-76.

MILGRAM, S.: Obedience to Autbority, Nueva York, Harper, 1974.

MIXON, D.: Understanding shocking and puzzling conduct en Ginsburg, G. P. Emerging Strategies in Social Psychological Research, Nueva York, Wiley, 1979.

ORNE, M.: On the Social Psychology of the psychological experiment: With particular reference to demand characteristics and their implications. American Psychologist, 1962, 17, 776-783.

PILIAVIN, I. M.; RODIN, J.; PILIAVIN, J. A.: Good Samaritanism: An Underground Phenomenom? Journal of Personality and Social Psycbology, 1969, 13, 289-299.

WICKER, A. W.: Ecological Psychology: some recent and prospective developments. American Psychologist, $1979,34,755.765$. 